\title{
Protocolo para Observação do Desenvolvimento Cognitivo e de Linguagem Expressiva (PODCLE)
}

\author{
Protocol for Expressive Language and Cognition Development \\ Observation (PELCDO)
}

\author{
Karina Elena Bernardis Bühler¹, Fabíola Custódio Flabiano², Suelly Cecilia Olivan Limongi², \\ Debora Maria Befi-Lopes ${ }^{4}$
}

\begin{abstract}
RESUMO
Objetivo: O objetivo do presente estudo foi a proposição de protocolo para observação do desenvolvimento cognitivo e de linguagem expressiva, referente ao período sensório-motor e início do pré-operatório, que pudesse ser utilizado como instrumento de análise objetiva das realizações observadas em crianças, possibilitando assim, a localização e acompanhamento das mesmas nesse processo de construção. Métodos: O Protocolo para Observação do Desenvolvimento Cognitivo e de Linguagem Expressiva (PODCLE) foi elaborado com base na literatura a respeito dos indicadores que caracterizam os estágios iniciais do desenvolvimento cognitivo e de linguagem expressiva, considerando-se o modelo proposto pela Epistemologia Genética. O Protocolo foi aplicado em crianças com desenvolvimento típico para verificação de sua viabilidade e levantamento de possíveis dúvidas ou dificuldades durante sua aplicação ou análise dos dados. Juízes foram utilizados para a seleção dos componentes deste Protocolo. Resultados: O Protocolo é constituído por quatro quadros contendo os indicadores do desenvolvimento cognitivo e de linguagem expressiva em seus estágios iniciais, bem como a pontuação correspondente às realizações apresentadas pela criança. Conclusão: O Protocolo para Observação do Desenvolvimento Cognitivo e de Linguagem Expressiva apresentado neste estudo é uma proposta de sistematização da observação de dados longitudinais referentes ao desenvolvimento cognitivo e de linguagem expressiva, durante o período sensório-motor e início do pré-operatório. Tal Protocolo permite, não apenas a localização, como também o acompanhamento da criança em seu processo de construção do conhecimento e da linguagem expressiva nos estágios iniciais do desenvolvimento.

Descritores: Cognição; Desenvolvimento da linguagem; Protocolos
\end{abstract}

\section{INTRODUÇÃO}

Seguindo o modelo teórico proposto pela Epistemologia Genética, cuja base está apoiada nos princípios propostos por

Trabalho realizado no Departamento de Fisioterapia, Fonoaudiologia e Terapia Ocupacional da Faculdade de Medicina da Universidade de São Paulo - USP - São Paulo (SP), Brasil, como parte de pesquisa financiada pela Fundação de Amparo à Pesquisa do Estado de São Paulo - FAPESP, em forma de Auxílio à Pesquisa.

(1) Pós-graduanda do Programa de Pós-Graduação em Ciências da Reabilitação da Faculdade de Medicina da Universidade de São Paulo - USP - São Paulo (SP), Brasil; Fonoaudióloga do Hospital Universitário da Universidade de São Paulo - HU-USP - São Paulo (SP), Brasil.

(2) Pesquisadora do Laboratório de Investigação Fonoaudiológica em Síndromes e Alterações Sensório-Motoras da Faculdade de Medicina da Universidade de São Paulo - USP - São Paulo (SP), Brasil; Pós-graduanda do Programa de Pós-Graduação em Ciências da Reabilitação da Faculdade de Medicina da Universidade de São Paulo - USP - São Paulo (SP), Brasil.

(3) Livre-Docente, Professora do Curso de Fonoaudiologia da Faculdade de Medicina da Universidade de São Paulo - USP - São Paulo (SP), Brasil.

(4) Livre-Docente, Professora do Curso de Fonoaudiologia da Faculdade de Medicina da Universidade de São Paulo - USP - São Paulo (SP), Brasil. Endereço para correspondência: Suelly Cecília Olivan Limongi. R. Cipotânia, 51, Cidade Universitária, São Paulo - SP, CEP 05360-160. E-mail: slimongi@usp.br

Recebido em: 30/10/2007; Aceito em: 28/2/2008
Piaget ${ }^{(1-3)}$, a linguagem possui relação direta, em seu desenvolvimento, com a construção do conhecimento, da cognição.

No início de vida, os bebês apresentam apenas ações reflexas. O constante exercício de tais ações, bem como sua coordenação e organização levam à construção de esquemas motores que, por sua vez, permitem a construção de esquemas mentais e, portanto, a condição de representação.

Nesse processo, a imitação desempenha papel fundamental, uma vez que revela a existência de condutas inteligentes, como o aprendizado da coordenação entre meios e fins. A imitação sensório-motora, que permite à criança imitar na presença do modelo, evolui para uma imitação que requer a representação mental, denominada imitação diferida. A partir desse momento, a criança torna-se capaz de diferenciar significantes de significados e começa a utilizar símbolos e signos, expressando-os por meio de gestos, sinais e/ou palavras ${ }^{(4.7)}$.

Piaget elaborou provas com o objetivo de identificar as estruturas subjacentes a um determinado comportamento. O método inicialmente empregado para tanto foi denominado de método clínico e propunha observar a própria ação do sujeito, mas sem prescindir de linguagem oral.

A partir das observações feitas por Piaget, foi realizado um estudo ${ }^{(8)}$ em que as provas e observações relacionadas ao 
período sensório-motor foram sistematizadas, com o objetivo maior de estudar o desenvolvimento da noção de permanência do objeto. Para tanto, utilizou-se do método clínico como proposto, mas com ênfase na observação da ação do sujeito sobre as tarefas solicitadas pelo observador, que faz uso tanto da própria ação quanto de intervenções orais para obter o desempenho solicitado.

O mesmo método serviu de base também para outro estu$\mathrm{do}^{(9)}$. Trata-se do método dialético-didático, assim denominado pela autora, que tem como objetivo construir um conhecimento comum entre o observador e o sujeito. O observador possui papel ativo na observação e analisará e avaliará os recursos cognitivos utilizados na realização da tarefa solicitada, o que é feito de forma dialética. Trata-se, portanto, de análise subjetiva, dependente da experiência e conhecimento do observador.

A preocupação em corroborar os princípios propostos pela Epistemologia Genética por meio de pesquisas que se valessem de estudos estatísticos motivou alguns pesquisadores do Grupo de Genebra a iniciarem tal busca, entre os anos 60 e $70^{(10-11)}$. Sem perder de vista a análise hierárquica do desenvolvimento cognitivo, a aplicação de técnicas analíticas visava descrições precisas enquanto eram mantidas a flexibilidade e a integridade do método clínico.

Em estudo complementar ${ }^{(12)}$, tal proposta foi sistematizada a partir da aplicação de uma prova operatória piagetiana, fornecendo informações importantes acerca da realização de seu objetivo: foi mantido o método de entrevista e consideradas tanto a ação concreta dos sujeitos sobre o material quanto a linguagem oral; foi utilizado o vídeo tape e posterior transcrição das situações de entrevista; foram estudados sujeitos cujas idades estavam tanto em plena fase do período considerado, quanto em fase de transição; no escore de desempenho, foram consideradas respostas dicotômicas do tipo sim/não, além de uma escala de valores que permitia a gradação das aquisições das habilidades. Segundo o autor, os resultados permitiram verificar a correspondência entre as estruturas hierárquicas do desenvolvimento cognitivo, o que foi corroborado pelo estudo estatístico realizado. Outros autores ${ }^{(13)}$ trabalharam com crianças que estavam no período sensório-motor e na transição para o pré-operatório e apontaram a importância da realização de análises quantitativas como comprovação e complementação de análises qualitativas.

Nas áreas de fala e linguagem especificamente abordadas em nosso país, alguns pesquisadores têm enfatizado a importância da mudança do paradigma relacionado à fundamentação do trabalho fonoaudiológico realizado, não mais baseado apenas na prática clínica, mas com base comprovada cientificamente ${ }^{(14-20)}$.

Para tanto, são citados testes padronizados traduzidos e adaptados para o português brasileiro, como também feita a proposição de protocolos. A utilização desses instrumentos vem justamente em concordância com a necessidade de favorecer análises e respostas objetivas às questões acima mencionadas.

Alguns estudos ${ }^{(21-22)}$, ao se referir à área da Fonoaudiologia no Brasil que considera os princípios propostos pela da Epistemologia Genética em sua aplicação, foram realizados com o objetivo de sistematização de dados qualitativos, embora sem apresentarem, ainda, propostas de quantificação.

Já outra pesquisa ${ }^{(23)}$, realizada em instituição nacional, utilizou proposta de sistematização de provas para avaliação cognitiva com a finalidade de relacioná-las com o desenvolvimento de linguagem. Nesse estudo, a análise qualitativa é partilhada com o objetivo de quantificação, uma vez que são atribuídos pontos para a manifestação de cada habilidade.

Em estudo recente ${ }^{(24)}$, autores observaram o processo de constituição da permanência do objeto em crianças durante o período sensório-motor, utilizando-se de análise qualitativa e quantitativa, por meio da atribuição de pontos de acordo com o comportamento apresentado pelos sujeitos. Foram encontrados resultados estatisticamente significantes, o que fortaleceu o estabelecimento do escore.

Nesse sentido, a utilização de protocolos que favoreçam a análise objetiva de dados qualitativos por meio da atribuição de pontos às realizações observadas referentes a indicadores de desenvolvimento se faz fundamental, visto que eles permitem a obtenção de escores com a localização dos sujeitos estudados em uma escala de valores, bem como a comparação com grupos-controle ou outros grupos-pesquisa ${ }^{(16-17,20,25-28)}$.

O objetivo do presente estudo foi a proposição do Protocolo para Observação do Desenvolvimento Cognitivo e de Linguagem Expressiva (PODCLE), referente ao período sensório-motor e início do pré-operatório, que permitisse uma análise objetiva das realizações e produções observadas em crianças, por meio do estabelecimento de indicadores e escores correspondentes, possibilitando, assim, a localização e acompanhamento da criança nesse processo de construção.

\section{MÉTODOS}

Este estudo foi aprovado pelo Comitê de Ética em Pesquisa do Hospital Universitário da Universidade de São Paulo, sob protocolo n ${ }^{\circ}$ 592/05 e pela Comissão de Ética para Análise de Projetos de Pesquisa CAPPesq da Diretoria Clínica do Hospital das Clínicas e da Faculdade de Medicina da Universidade de São Paulo, sob protocolo $n^{\circ}$ 082/07.

O PODCLE foi elaborado com base na literatura ${ }^{(1-3,29-32)}$. Tais estudos foram tomados como referências para a sistematização dos indicadores que caracterizam o período sensóriomotor e início do pré-operatório, tanto do ponto de vista do desenvolvimento cognitivo quanto da linguagem expressiva. As aquisições foram organizadas de forma a obedecer à hierarquia do processo de construção, como propõem os referidos autores.

O PODCLE foi organizado em quadros que contêm, separadamente, os indicadores referentes ao desenvolvimento cognitivo e ao de linguagem expressiva. A proposta para análise dos dados referentes às realizações e produções das crianças permite não apenas a localização da criança em seu desenvolvimento cognitivo e de linguagem expressiva, mas também o seu acompanhamento durante esses estágios iniciais.

Tal fato é possível uma vez que, como preconiza a Epistemologia Genética, a construção do conhecimento é um processo hierárquico e cumulativo e sua sistematização favorece a adoção de indicadores de desenvolvimento representados pelas próprias realizações e produções das crianças. 
Para a análise são considerados pontos de acordo com as realizações apresentadas pelas crianças, relacionadas a cada um dos indicadores característicos do período estudado, cuja soma indica a localização da criança em seu desenvolvimento.

O presente Protocolo foi aplicado como estudo piloto ${ }^{(33)} \mathrm{em}$ cinco crianças com desenvolvimento típico, que freqüentavam a creche do Hospital Universitário da USP, acompanhadas mensalmente durante 12 meses. Para tanto, foram utilizados dois juizes, fonoaudiólogas com experiência na área e na aplicação do método dialético-didático, sendo uma doutora e uma doutoranda. Tal procedimento foi realizado visando a seleção dos indicadores de desenvolvimento cognitivo e de linguagem expressiva que comporiam o Protocolo, bem como a verificação de sua viabilidade e o levantamento de possíveis dúvidas ou dificuldades durante sua aplicação e/ou análise dos dados.

As fonoaudiólogas confirmaram que o PODCLE foi de fácil aplicação e interpretação. A duração média para aplicação do Protocolo foi de 20 minutos e, para a transcrição e análise dos dados, as fonoaudiólogas levaram de 30 a 60 minutos, dependendo da idade da criança. A concordância em relação aos indicadores de desenvolvimento cognitivo e de linguagem expressiva que compõem o presente protocolo foi de $93 \%$ para o juiz 1 e de $94 \%$ para o juiz 2.

\section{RESULTADOS}

Como resultado, propõe-se o Protocolo para Observação do Desenvolvimento Cognitivo e de Linguagem Expressiva (Anexo 1, Quadros 1, 2, 3 e 4), referente ao período sensóriomotor e início do pré-operatório.

O PODCLE é composto de uma folha para descrição das observações em relação ao desempenho da criança (Anexo 1) e quatro quadros referentes à pontuação correspondente (Quadros 1, 2, 3 e 4).

O Quadro 1 refere-se à atribuição de pontos de acordo com as realizações da criança em relação aos indicadores de desenvolvimento cognitivo durante o período sensório-motor e início do pré-operatório.

Os indicadores que compõem o Quadro 1, bem como os procedimentos para sua verificação são apresentados a seguir:

1. Aplicação de esquemas sensório-motores: oferecer à criança um objeto de seu interesse (um bichinho de borracha e/ou um chocalho), deixando-a explorá-lo por dois minutos. Deve ser observado: quais esquemas motores isolados (por exemplo: pegar, chacoalhar, bater, olhar, chupar) e/ou coordenados (por exemplo: pegar-olhar e levar à boca, olhar-agarrar e

Quadro 1. Protocolo de pontuação da cognição

\begin{tabular}{|c|c|c|c|c|c|}
\hline \multicolumn{6}{|c|}{ Pontuação } \\
\hline \multirow{2}{*}{$\begin{array}{l}\text { Indicadores do } \\
\text { Desenvolvimento } \\
\text { Cognitivo }\end{array}$} & \multicolumn{5}{|c|}{ Realizações da Criança } \\
\hline & 0 & 1 & 2 & 3 & 4 \\
\hline $\begin{array}{c}\text { Aplicação de } \\
\text { esquemas sensório- } \\
\text { motores }\end{array}$ & não realiza & $\begin{array}{c}\text { aplicação de } \\
\text { esquemas isolados }\end{array}$ & $\begin{array}{l}\text { aplicação de } \\
\text { esquemas } \\
\text { coordenados }\end{array}$ & & \\
\hline $\begin{array}{l}\text { Deslocamento dos } \\
\text { objetos no espaço }\end{array}$ & não realiza & $\begin{array}{l}\text { acompanha de forma } \\
\text { incompleta }\end{array}$ & $\begin{array}{c}\text { acompanha de forma } \\
\text { completa }\end{array}$ & & \\
\hline $\begin{array}{c}\text { Permanência do } \\
\text { objeto }\end{array}$ & não realiza & $\begin{array}{l}\text { procura pelo objeto } \\
\text { parcialmente } \\
\text { escondido }\end{array}$ & $\begin{array}{l}\text { procura pelo objeto } \\
\text { totalmente escondido }\end{array}$ & $\begin{array}{c}\text { realiza considerando } \\
\text { deslocamentos } \\
\text { visíveis }\end{array}$ & $\begin{array}{c}\text { realiza considerando } \\
\text { deslocamentos } \\
\text { invisíveis }\end{array}$ \\
\hline \multicolumn{6}{|l|}{$\begin{array}{c}\text { Imitação de } \\
\text { esquemas motores }\end{array}$} \\
\hline (com o modelo) & não realiza & $\begin{array}{l}\text { esquemas isolados } \\
\text { visíveis no próprio } \\
\text { corpo }\end{array}$ & $\begin{array}{l}\text { esquemas isolados } \\
\text { não visíveis no } \\
\text { próprio corpo }\end{array}$ & $\begin{array}{c}\text { esquemas que } \\
\text { designam função aos } \\
\text { objetos }\end{array}$ & \\
\hline $\begin{array}{l}\text { Experiências com } \\
\text { objetos novos }\end{array}$ & não realiza & & realiza & & \\
\hline $\begin{array}{l}\text { Uso de objeto como } \\
\text { meio }\end{array}$ & não realiza & conduta do suporte & conduta do barbante & conduta da vara & \\
\hline $\begin{array}{l}\text { Uso de esquemas } \\
\text { simbólicos simples } \\
\text { (sem o modelo) }\end{array}$ & não realiza & $\begin{array}{c}\text { aplica no próprio } \\
\text { corpo }\end{array}$ & $\begin{array}{l}\text { aplica em objetos } \\
\text { figurativos }\end{array}$ & $\begin{array}{l}\text { aplica em objetos } \\
\text { não figurativos }\end{array}$ & \\
\hline $\begin{array}{l}\text { Uso de esquemas } \\
\text { simbólicos } \\
\text { combinados }\end{array}$ & não realiza & combina duas ações & $\begin{array}{l}\text { combina três ou } \\
\text { mais ações não } \\
\text { ordenadas }\end{array}$ & $\begin{array}{l}\text { combina três ou mais } \\
\text { ações ordenadas }\end{array}$ & \\
\hline
\end{tabular}


Quadro 2. Protocolo de pontuação da linguagem expressiva

\begin{tabular}{|c|c|c|c|c|c|c|c|}
\hline \multicolumn{8}{|c|}{ Pontuação } \\
\hline \multirow{2}{*}{$\begin{array}{l}\text { Indicadores do } \\
\text { Desenvolvimento } \\
\text { de Linguagem } \\
\text { Expressiva }\end{array}$} & \multicolumn{7}{|c|}{ Produções da Criança } \\
\hline & 0 & 1 & 2 & 3 & 4 & 5 & 6 \\
\hline $\begin{array}{l}\text { uso de gestos } \\
\text { dêiticos }\end{array}$ & não realiza & $\begin{array}{c}\text { por imitação } \\
\text { simples (com o } \\
\text { modelo) }\end{array}$ & $\begin{array}{c}\text { por imitação } \\
\text { simples } \\
\text { acompanhados } \\
\text { de } \\
\text { vocalizações }\end{array}$ & $\begin{array}{c}\text { por imitação } \\
\text { diferida (sem o } \\
\text { modelo) }\end{array}$ & $\begin{array}{c}\text { por imitação } \\
\text { diferida } \\
\text { acompanhados } \\
\text { de } \\
\text { vocalizações }\end{array}$ & & \\
\hline $\begin{array}{l}\text { uso de gestos } \\
\text { representativos }\end{array}$ & não realiza & $\begin{array}{c}\text { por imitação } \\
\text { simples (com o } \\
\text { modelo) }\end{array}$ & $\begin{array}{c}\text { por imitação } \\
\text { simples } \\
\text { acompanhados } \\
\text { de } \\
\text { vocalizações }\end{array}$ & $\begin{array}{l}\text { por imitação } \\
\text { diferida (sem } \\
\text { o modelo) ou } \\
\text { representação } \\
\text { simbólica }\end{array}$ & $\begin{array}{c}\text { por imitação } \\
\text { diferida ou } \\
\text { representação } \\
\text { simbólica, } \\
\text { acompanhados } \\
\text { de } \\
\text { vocalizações }\end{array}$ & $\begin{array}{c}\text { por imitação } \\
\text { diferida, } \\
\text { acompanhados } \\
\text { de sílabas com } \\
\text { significado ou } \\
\text { onomatopéias } \\
\text { vocais }\end{array}$ & \\
\hline $\begin{array}{c}\text { produção de } \\
\text { verbalizações } \\
\text { acompanhadas } \\
\text { por gestos }\end{array}$ & não realiza & $\begin{array}{l}\text { sílabas com } \\
\text { significado }\end{array}$ & $\begin{array}{c}\text { palavras } \\
\text { monossilábicas } \\
\text { e/ou } \\
\text { interjeições }\end{array}$ & $\begin{array}{c}\text { palavras } \\
\text { onomatopaicas }\end{array}$ & $\begin{array}{c}\text { palavras } \\
\text { isoladas com } \\
\text { mais de uma } \\
\text { sílaba }\end{array}$ & $\begin{array}{l}\text { combinação de } \\
\text { duas palavras }\end{array}$ & $\begin{array}{c}\text { combinação de } \\
\text { mais de duas } \\
\text { palavras }\end{array}$ \\
\hline $\begin{array}{c}\text { produção de } \\
\text { verbalizações } \\
\text { isoladas }\end{array}$ & não realiza & $\begin{array}{c}\text { sílabas com } \\
\text { significado } \\
\text { relacionadas } \\
\text { ao contexto ou } \\
\text { objeto }\end{array}$ & $\begin{array}{c}\text { palavras } \\
\text { monossilábicas } \\
\text { e/ou } \\
\text { interjeições }\end{array}$ & $\begin{array}{c}\text { palavras } \\
\text { onomatopaicas }\end{array}$ & $\begin{array}{c}\text { palavras } \\
\text { isoladas com } \\
\text { mais de uma } \\
\text { sílaba }\end{array}$ & $\begin{array}{l}\text { combinação de } \\
\text { duas palavras }\end{array}$ & $\begin{array}{c}\text { combinação de } \\
\text { mais de duas } \\
\text { palavras }\end{array}$ \\
\hline
\end{tabular}

Quadro 3. Protocolo de pontuação da cognição e da linguagem expressiva

\begin{tabular}{|c|c|c|c|}
\hline Cognição & Pontuação & Linguagem Expressiva & Pontuação \\
\hline $\begin{array}{c}\text { Aplicação de esquemas } \\
\text { sensório-motores }\end{array}$ & Uso de gestos dêiticos & Uso de gestos representativos \\
\hline $\begin{array}{c}\text { Deslocamento dos objetos no } \\
\text { espaço }\end{array}$ & Produção de verbalizações & acompanhadas por gestos & \\
\hline $\begin{array}{c}\text { Permanência do objeto } \\
\text { (com o modelo) }\end{array}$ & Produção de verbalizações & isoladas & \\
\hline $\begin{array}{c}\text { Imitação de esquemas motores } \\
\text { Experiências com objetos novos }\end{array}$ & & & \\
\hline Uso de objetos como meio & & & \\
\hline $\begin{array}{c}\text { Uso de esquemas simbólicos } \\
\text { simples (sem o modelo) }\end{array}$ & & & \\
\hline $\begin{array}{c}\text { Uso de esquemas simbólicos } \\
\text { combinados }\end{array}$ & & & \\
\hline TOTAL & & & \\
\hline
\end{tabular}


Quadro 4. Quadro de referência para as fases do desenvolvimento cognitivo (período sensório motor e início do pré-operatório)

\begin{tabular}{|c|c|c|}
\hline Pontuação & Realizações & Fase do desenvolvimento cognitivo \\
\hline 0 a 3 pontos & $\begin{array}{l}\text { Uso de esquemas isolados e acompanhamento do deslocamento do objeto } \\
\text { no espaço }\end{array}$ & $\begin{array}{c}1^{\mathrm{a}} \text { e } 2^{\mathrm{a}} \text {. fases do Período Sensório- } \\
\text { Motor }\end{array}$ \\
\hline 3,1 a 6 pontos & $\begin{array}{l}\text { Uso de esquemas coordenados, procura pelo objeto parcialmente escondido, } \\
\text { imitação de esquemas visíveis no próprio corpo }\end{array}$ & $3^{\mathrm{a}}$. fase do Período Sensório-Motor \\
\hline 6,1 a 10 pontos & $\begin{array}{l}\text { Procura pelo objeto totalmente escondido, mas sem considerar seus } \\
\text { deslocamentos, imitação de esquemas não visíveis no próprio corpo, } \\
\text { condutas do suporte e do barbante }\end{array}$ & $4^{a}$. fase do Período Sensório-Motor \\
\hline 10,1 a 15 pontos & $\begin{array}{l}\text { Procura pelo objeto totalmente escondido, considerando apenas os } \\
\text { deslocamentos visíveis, imitação de esquemas coordenados, realiza } \\
\text { experiências com objetos novos e a conduta da vara }\end{array}$ & $5^{\text {a }}$. fase do Período Sensório-Motor \\
\hline 15,1 a 18 pontos & $\begin{array}{l}\text { Procura pelo objeto totalmente escondido, considerando os deslocamentos } \\
\text { visíveis e invisíveis, Uso de esquemas simbólicos isolados, aplicados no } \\
\text { próprio corpo ou em objetos figurativos }\end{array}$ & $6^{a}$. fase do Período Sensório-Motor \\
\hline 18,1 a 22 pontos & $\begin{array}{c}\text { Uso de esquemas simbólicos aplicados em material não figurativo, uso de } \\
\text { esquemas simbólicos combinados. }\end{array}$ & Início do Período Pré-Operatório. \\
\hline
\end{tabular}

chacoalhar, passar o objeto de uma mão para outra) a criança aplica ao objeto.

2. Deslocamento dos objetos no espaço: apresentar à criança um objeto de seu interesse (um bichinho de borracha e/ou um chocalho), em linha média e na altura dos olhos. Chamar a atenção da criança para o objeto. Em seguida, deslocar o objeto do centro para a direita e da direita para a esquerda, retornando ao centro devagar, uma única vez. Deve ser observado: se a criança é capaz de acompanhar visualmente o deslocamento lateral do objeto de forma incompleta (quando a criança em algum momento deixa de acompanhar a trajetória de deslocamento do objeto, desviando o olhar) ou de forma completa.

\section{Permanência do objeto:}

3.1. Apresentar à criança um objeto de seu interesse (boneca, carrinho, bola ou chocalho), o qual deve ser totalmente escondido sob um lenço A. Deve ser observado: procura e preensão do objeto totalmente escondido. Caso a criança não demonstre intenção de puxar o lenço para pegar o objeto, o examinador deve deixar uma pequena parte do objeto descoberto. Se a criança obtiver sucesso com o objeto parcialmente escondido, tentar novamente com o objeto totalmente escondido. Se a criança obtiver sucesso com o objeto totalmente escondido, aplicar o item 3.2. Caso contrário a prova é finalizada.

3.2. Reapresentar à criança o objeto de seu interesse e introduzir um segundo lenço B (de cor diferente). Esconder o objeto de interesse da criança sob o lenço A e, depois que ela o reencontrar, deslocá-lo às vistas da criança para o lenço B. Deve ser observado: comportamento da criança na procura do objeto ausente (procura apenas em A, procura primeiro em $\mathrm{A}$ e depois em $\mathrm{B}$, procura direto em $\mathrm{B}$, ou outro comportamento). Se a criança for capaz de considerar os deslocamentos visíveis dos objetos, procurando diretamente em B, aplicar o item 3.3. Caso contrário, a prova é finalizada.

3.3. Apresentar à criança um objeto pequeno de seu interes- se que possa ser escondido na mão fechada do examinador. Esconder o objeto na mão do examinador às vistas da criança e deslocá-lo com a mão fechada para outro local (por exemplo: dentro do bolso, embaixo do colchonete, sob um lenço ou sob outro objeto), de forma que o deslocamento do objeto seja realizado de forma invisível para a criança. Deve ser observado: comportamento da criança na procura do objeto escondido (procura diretamente no local onde o objeto foi escondido após seu deslocamento invisível, procura na mão do examinador ou em outro lugar, ou apresenta outro comportamento).

Estes procedimentos devem ser aplicados por três vezes consecutivas para cada item, sendo considerada a resposta de maior ocorrência.

4. Imitação de esquemas motores: devem ser oferecidos pelo examinador os seguintes modelos de esquemas motores para imitação:

a) esquemas visíveis no próprio corpo: bater palmas e dar tchau.

b) esquemas não visíveis no próprio corpo: mandar beijo com estalos de lábios e realizar vibração de lábios.

c) esquemas que designam funções aos objetos: levar o telefone à orelha e empurrar o carrinho.

Deve ser considerada, para fins de análise, a imitação de outros esquemas motores, diferentes dos esquemas citados acima, que tenham sido imediatamente reproduzidos pela criança durante a sessão de observação.

$\mathrm{O}$ examinador deve garantir que a criança mantenha a atenção no momento da apresentação do modelo.

5. Experiências com objetos novos: referem-se às reações circulares terciárias, observadas por meio de experiências com objetos desconhecidos da criança.

Deve ser oferecido para a criança uma caixa de sapato tampada, ou um pote de plástico transparente com tampa de rosquear, ambos contendo peças de encaixe tipo "lego", e uma caixa de fósforos com clipes de papel. A criança poderá 
explorá-los livremente durante 5 minutos. Deve ser observado: quais esquemas são utilizados para explorar o objeto de modo a identificar um objetivo na ação da criança (por exemplo: se aplica os esquemas de bater, jogar, pressionar a tampa, variando a intensidade da força empregada para abrir o pote; se empurra o pote para que role sobre a superfície, variando a intensidade da força aplicada, observando os efeitos visuais de suas ações no objeto).

6. Uso de objetos como meios: referem-se às condutas do suporte do barbante e da vara.

a. Apresentar um objeto de interesse da criança (bichinho de borracha, chocalho) sobre a extremidade distante de um lenço, deixando apenas a extremidade oposta ao alcance da criança. A examinadora deve chamar a atenção da criança, apontando ou tocando o objeto por no máximo três vezes. Deve ser observado: se a criança usa o lenço (puxa-o) como um meio para trazer para si o objeto de interesse (conduta do suporte).

b. Apresentar para a criança um carrinho amarrado a um barbante, de forma que o carrinho fique inacessível e o barbante ao alcance da criança. A examinadora deve chamar a atenção da criança para o carrinho, por no máximo três vezes. Deve ser observado: se a criança utiliza o barbante para puxar o carrinho para si (conduta do barbante) ou se tenta pegá-lo diretamente.

c. Colocar fora do alcance da criança um objeto de seu interesse (caixinha de fósforos, bola, peça de encaixe tipo "lego") e apresentar-lhe um objeto longo (vara ou coçador de costas) ao alcance de suas mãos. A examinadora deve chamar a atenção para o objeto de interesse por no máximo três vezes. Deve ser observado: se a criança tenta pegar o objeto diretamente, se usa a vara como instrumento para trazer o objeto para perto de si (conduta da vara) ou apresenta outro comportamento.

7. Uso de esquemas simbólicos simples: devem ser entregues à criança materiais figurativos (uma boneca ou urso de pelúcia, um telefone de brinquedo, um carrinho, uma banheira, uma colher ou garfo, um prato, um copo, uma panela, dois potes pequenos com tampa) e não figurativos (blocos lógicos). Deve ser observado: se a criança utiliza esquemas simbólicos isolados, como, por exemplo, levar o telefone à orelha ou levar o garfo à sua boca (aplicação de esquemas simbólicos no próprio corpo); levar a colher à boca da boneca ou empurrar o carrinho (aplicação de esquemas simbólicos com objetos figurativos); levar um bloco lógico à própria boca ou à boca da boneca, utilizando-o como alimento ou esfregar um bloco lógico no corpo da boneca, utilizando-o como sabonete (aplicação de esquemas simbólicos com objetos não-figurativos). Observar se a criança os realiza de maneira espontânea, na ausência do modelo, o que caracteriza a imitação diferida.

8. Uso de esquemas simbólicos combinados: deve ser verificado durante a utilização dos mesmos materiais descritos no item 7. Deve ser observado: se a criança realiza combinação de apenas dois esquemas simbólicos, por exemplo: mexe a colher dentro de um potinho e oferece à boneca em seguida ou aperta as teclas do telefone, levando o fone à orelha em seguida, ou se realiza mais de dois esquemas combinados. Caso a criança utilize combinação de mais de dois esquemas simbólicos, observar se apresentam ou não ordenação temporal. Por exemplo, a criança coloca a boneca de roupa na banheira, passa o sabonete e depois tira a roupa (combinação de mais de dois esquemas simbólicos não ordenados), ou se a criança tira a roupa da boneca, coloca-a na banheira, passa o sabonete e finge enxaguá-la em seguida (combinação de mais de dois esquemas simbólicos ordenados)

O Quadro 2 refere-se à atribuição de pontos de acordo com as produções apresentadas em relação aos indicadores do desenvolvimento de linguagem expressiva durante o período sensório-motor e início do pré-operatório. Os indicadores componentes do Quadro 2, bem como os procedimentos para verificação dos mesmos, são apresentados a seguir:

1. Uso de gestos dêiticos: os gestos dêiticos são aqueles que têm a função de designação, com a intenção de por algo ou algum evento em evidência ${ }^{(31)}$. Deve ser observado: uso de gestos dêiticos realizados por imitação simples (na presença do modelo), acompanhados ou não por vocalizações, e gestos dêiticos realizados por imitação diferida (sem a presença do modelo), acompanhados ou não por vocalizações. São exemplos de gestos dêiticos: gesto de apontar, gesto de estender o braço ou a mão para o objeto de desejo, gesto de chamar (abrindo e fechando a mão).

2. Uso de gestos representativos: os gestos representativos são aqueles que favorecem a capacidade de evocação de objetos, que exprimem a globalidade e têm função referencial ${ }^{(31)}$. Deve ser observado: uso de gestos representativos (por exemplo: mandar beijos, dar tchau, colocar o dedo indicador em frente à boca - silêncio -, movimentar o dedo indicador em sinal de "não") realizados por imitação simples (na presença do modelo), acompanhados ou não por vocalizações; uso de gestos representativos realizados por imitação diferida (sem a presença do modelo) ou representação simbólica, gestos acompanhados ou não por vocalizações desprovidas de significado, uso de gestos representativos realizados por imitação diferida, acompanhados por sílabas com significado (quando a sílaba acompanha o objeto, como por exemplo: leva o telefone à orelha e produz a sílaba com significado /lô/ [alô], nina a boneca e emite a sílaba com significado /ne/ [nenê] ou onomatopéias vocais, por exemplo: gesto representativo de empurrar um carrinho acompanhado de vibração de lábios, [onomatopéia vocal "vumm vummmm"] ou de levar o garfo à boca, realizando estalos de língua [onomatopéia vocal]).

3. Produção de verbalizações acompanhadas por gestos: deve ser observada a produção dos seguintes itens:

3.1. Produção de sílabas com significado, relacionadas ao contexto ou objeto (por exemplo: produção de "ó" (olha), acompanhado do gesto de apontar, da sílaba com significado "bô", acompanhada do gesto de "acabou", virando as palmas das mãos para cima e abrindo os braços).

3.2. Produção de palavras isoladas:

- palavras monossilábicas elou interjeições, como por 
exemplo: produção da palavra "é", acompanhada do gesto representativo de balançar a cabeça em sinal de "sim"; "lá" acompanhada do gesto de apontar; "aô" (alô), acompanhada do gesto de levar o telefone à orelha, "tau" (tchau), acompanhada do gesto de dar tchau, etc. Diferenciam-se das sílabas com significado, acompanhadas por gestos dêiticos ou representativos, descritas no item 3.1, por constituírem palavras monossilábicas ou interjeições, segundo a gramática da língua portuguesa.

- palavras onomatopaicas, considerando-se as que não são dicionarizadas (por exemplo: "papá", utilizado como substantivo acompanhado do gesto de apontar, "bibi" acompanhado do gesto de empurrar o carrinho).

- palavras isoladas com mais de uma sílaba: exemplo: "vovó" e "papai", acompanhadas do gesto de levar o telefone à orelha, "binquedo" (brinquedo) acompanhado do gesto dêitico de estender o braço, pedindo a sacola de brinquedos para a examinadora.

3.3. Produção de palavras combinadas: (combinação de duas ou mais palavras), como por exemplo: combinações de duas palavras "o oio" (o olho), ao apontar para o olho da boneca; "abô eti" (acabou o sabonete) acompanhado do gesto de acabou; "qué bê i dentu" (quero ver aí dentro), apontando para a sacola de brinquedos. Ressalta-se que não devem ser considerados os processos fonológicos produzidos pelas crianças.

4. Produção de verbalizações isoladas: deve ser observada a produção dos seguintes itens: produção de sílabas com significado, relacionadas ao contexto ou objeto, produção de palavras isoladas (palavras monossilábicas e/ou interjeições, palavras onomatopaicas, palavras isoladas com mais de uma sílaba), ou combinadas (combinação de duas ou mais palavras), produzidas de forma isolada, ou seja, não acompanhadas de gestos, porém contextualizadas.

O Quadro 3 apresenta a síntese da pontuação obtida para os indicadores do desenvolvimento cognitivo e de linguagem expressiva, cuja soma indica a localização da criança em seu desenvolvimento.

O Quadro 4 apresenta as referências que caracterizam as fases do desenvolvimento cognitivo relacionadas ao período sensório-motor e pré-operatório.

\section{Considerações sobre a aplicação do Protocolo}

Para a aplicação do PODCLE, é necessário que o material utilizado seja constituído por brinquedos que fazem parte do universo da criança nos dois primeiros anos de vida: bichinho de borracha, chocalho, boneca, carrinho, bola, caixa vazia de fósforos com clipes de papel dentro, caixa de sapato com tampa, pote de plástico transparente com tampa de rosca, peças coloridas tipo "lego", vara ou coçador de costas, telefone, banheirinha, colher ou garfo, prato, copo, panela, blocos lógicos e dois lenços de cores diferentes.

Deve-se considerar, também, a utilização de uma filmadora.

É importante que a situação de observação seja realizada com a criança em estado alerta e confortável e que toda a sessão seja gravada em vídeo, para posterior transcrição e preenchimento dos quadros garantindo, assim, maior fidedignidade e precisão dos dados observados.

Os indicadores referentes ao desenvolvimento cognitivo devem ser verificados na ordem em que os procedimentos são apresentados no Protocolo. Os indicadores em relação ao desenvolvimento de linguagem expressiva devem ser verificados durante a aplicação das provas cognitivas. Não devem ser consideradas, para fins de análise dos dados, as informações trazidas pelos pais ou cuidadores a respeito do desenvolvimento cognitivo e de linguagem que não sejam observadas durante a aplicação do Protocolo.

O presente Protocolo pode ser utilizado com dois objetivos:

1) localizar a criança em seu desenvolvimento cognitivo e de linguagem expressiva com relação aos estágios iniciais;

2) acompanhar o desenvolvimento da criança longitudinalmente, ou seja, em diferentes momentos ao longo de seu processo de construção cognitiva e de linguagem expressiva, durante o período sensório-motor e início do préoperatório.

No caso da observação longitudinal, os dados observados devem ser considerados como parte de um processo contínuo em que as aquisições são cumulativas. Assim, a pontuação obtida em sessões anteriores, em que não haja dúvida do que foi observado, deverá ser mantida para a próxima sessão, caso não seja possível observar determinado indicador novamente.

\section{DISCUSSÃO}

A proposta para a formalização dos indicadores de desenvolvimento cognitivo e de linguagem expressiva apresentados no presente Protocolo foi determinada após o estudo e a sistematização das realizações e produções características do período sensório-motor e pré-operatório, tomando-se como base os pressupostos da Epistemologia Genética ${ }^{(1-3)}$ e estudos de autores que partilham a mesma base teórica ${ }^{(29-32)}$.

A determinação de pontos relacionados às respostas dadas de acordo com a realização de atividades tem como objetivo o estabelecimento de valores que poderão identificar parâmetros e indicadores - no caso do presente Protocolo proposto - referentes ao processo de desenvolvimento cognitivo e de linguagem expressiva, possibilitando a localização da criança no processo de desenvolvimento em que se encontra. Isto é possível uma vez que o modelo teórico que fornece o substrato para o estudo desse desenvolvimento é pautado em pressupostos de hierarquia na construção do conhecimento e no fator cumulativo do mesmo ${ }^{(1-3)}$.

Estudos em outras áreas enfatizam a importância na utilização de pontos, também com o objetivo de fornecer escores e parâmetros para o controle objetivo e sistemático de tratamentos ou processo terapêuticos, além de permitirem a comparação dos resultados com os de grupos-controle ou outros grupos-pesquisa ${ }^{(16-17,25-28)}$.

A Fonoaudiologia necessita cumprir a proposição de trabalhar com avaliações e tratamentos tendo como base as evidências, de tal forma que os procedimentos e técnicas utilizados sejam fundamentados em pesquisas cientificamente 
estruturadas ${ }^{(34)}$. As ações baseadas em evidências favorecem, não apenas maior adequação em procedimentos de avaliação e diagnóstico, como também nos relacionados aos tratamentos e reavaliações e indicam, de forma mais precisa, o melhor caminho a ser seguido, tanto na continuação de uma determinada intervenção, quanto em possíveis mudanças ${ }^{(25-28)}$.

A utilização do método dialético-didático ${ }^{(9)}$ para a obtenção dos dados necessários à análise qualitativa quantificada ${ }^{(10-12)}$ do desenvolvimento de crianças no período sensório-motor e início do pré-operatório, mostrou-se uma escolha acertada, encontrando respaldo em estudo realizado em instituição internacional ${ }^{(13)}$, uma vez que tornou possível aliar a observação qualitativa do sujeito em seu processo de desenvolvimento à análise objetiva de tais dados, permitindo o uso de tratamento estatístico.

Faz-se necessário, portanto, que o profissional fonoaudiólogo utilize procedimentos mais objetivos de avaliação e tratamento, considerando os dados não apenas de forma qualitativa e sim quantificando as análises qualitativas. Dessa forma, será possível, a partir dos marcos referenciais fornecidos pelo modelo teórico, que caracterizam os indicadores de cada fase de desenvolvimento, sistematizar parâmetros para identificação e localização dos sujeitos no percurso do processo de construção cognitiva e de linguagem expressiva.

\section{CONCLUSÃo}

O Protocolo para Observação do Desenvolvimento Cognitivo e de Linguagem Expressiva (PODCLE), proposto no presente estudo, consiste em instrumento que permite ao profissional interessado no estudo do desenvolvimento de linguagem e cognição em crianças pequenas, localizar a criança nesse processo de construção, bem como acompanhar o seu desenvolvimento de forma objetiva. Tal Protocolo pode ser aplicado em diferentes populações e permite a comparação entre grupos pesquisa e controle, contribuindo assim, para a sistematização de estudos na área.

\section{AGRADECIMENTOS}

Agradecemos à Dra. Karina de Araújo e às fonoaudiólogas Isabela Gomes e Marina Leite Puglisi pelas importantes considerações, que contribuíram para a estruturação deste protocolo.

\begin{abstract}
Purpose: The aim of the present study was to propose a protocol for the observation of expressive language and cognition development during sensorimotor and the beginning of pre-operatory periods that could be used as a tool for the objective analysis of children's productions, allowing the localization and follow-up of these children during this construction process. Methods: The Protocol for Expressive Language and Cognition Development Observation (PELCDO) was based on literature concerning the indicators of early expressive language and cognition development. The PELCDO was applied in typically developing children, with the aim to verify its viability and to identify possible doubts or difficulties during its management or data analysis. Judges were used for the selection of the indicators of cognitive and expressive language development. Results: The Protocol has four tables that comprise the indicators of early expressive language and cognition development, as well as its related score, according to the child's performance. Conclusion: The Protocol presented in this study is an attempt to systematize the observation of longitudinal data regarding language and cognition development, during the sensorimotor and beginning of pre-operatory periods. Such Protocol not only allows the child's localization, but also makes possible to follow the individual along the knowledge and language construction process on these early developmental stages.
\end{abstract}

Keywords: Cognition; Language development; Protocols

\title{
REFERÊNCIAS
}

1. Piaget J. Construção do real na criança. Rio de Janeiro: Zahar Editora; 1970. [edição original 1937].

2. Piaget J. A formação do símbolo na criança: imitação, jogo e sonho, imagem e representação. 3a ed. Rio de Janeiro: Zahar; 1978. [edição original 1946].

3. Piaget J. Nascimento da inteligência na criança. 3a ed. Rio de Janeiro: Zahar; 1978. [edição original 1936].

4. McCarty ME. Actions with tools. Enfance. 2000;52(3):249-57.

5 . Bremner JG. The nature of imitation by infants. Infant Behav Dev. 2002;25(1):65-7.

6. Iverson JM, Goldin-Meadow S. Gesture paves the way for language development. Psychol Sci. 2005;16(5):367-71.

7. Ozçaliskan S, Goldin-Meadow S. Gesture is at the cutting edge of early language development. Cognition. 2005;96(3):B101-13
8. Carraher TN. Método clínico: usando os exames de Piaget. Petrópolis: Vozes; 1983.

9. Parrat-Dayan S. Processos internos y externos en la construcción de una explicación causal. In : Assis MC, Assis OZM, Ramozzi-Chiarottino Z, organizadores. Piaget: teoria e prática: centenário de Jean Piaget 1896-1996. Campinas: Tecnocopias Gráficas e Editora; 1996. p 58-68.

10. Bond TG. Piaget and measurement I: the twain really do meet. Arch Psychol. 1995;63:71-87.

11. Bond TG. Piaget and measurement II: empirical validation of the Piagetian model. Arch Psychol. 1995;63:155-85.

12. Bond TG. Piaget and measurement III: Reassessing the Méthode Clinique. Arch Psychol. 1995;63:231-55.

13. Adrien JL, Rossignol-Deletang N, Martineau J, Couturier G, Barthelemy C. Regulation of cognitive activity and early communication 
development in young autistic, mentally retarded, and young normal children. Dev Psychobiol. 2001;39(2):124-36.

14. Neiva FC, Wertzner HF. A protocol for oral myofunctional assessment: for application with children. Int J Orofacial Myology. 1996;22:8-19.

15. Andrade CRF, Befi-Lopes DM, Fernandes FDM, Wertzner HF, organizadores. ABFW: teste de linguagem infantil nas áreas de fonologia, vocabulário, fluência e pragmática. 2a ed. Barueri: Pró-Fono Departamento Editorial; 2004.

16. Andrade CRF, Juste F. Proposta de análise de performance e de evolução em crianças com gagueira desenvolvimental. Rev CEFAC. 2005;7(2):158-70.

17. Trindade IEK, Genaro KF, Yamashita RP, Miguel HC, Fukushiro AP. Proposta de classificação da função velofaríngea na avaliação perceptivo-auditiva da fala. Pró-Fono. 2005;17(2):259-62.

18. Giusti E. Performance de crianças falantes do português brasileiro no Test of Early Language Development (TELD-3) [tese]. São Paulo: Faculdade de Filosofia, Letras e Ciências Humanas da Universidade de São Paulo; 2007.

19. Padovani AR, Moraes DP, Mangili LD, Andrade CRF. Protocolo Fonoaudiológico de Avaliação do Risco para Disfagia (PARD). Rev Soc Bras Fonoaudiol. 2007;12(3):199-205.

20. Befi-Lopes DM, Takiuchi N, Araújo K. Maturidade simbólica e linguagem: estudo follow-up. In: Sociedade Brasileira de Fonoaudiologia. Atualização em Voz, Linguagem, Audição e Motricidade Oral. São Paulo: Frontis; 2000. p.1-16.

21. Limongi SCO, Carvallo RMM, Souza ER. Auditory processing and language in Down syndrome. J Med Speech Lang Pathol. 2000;8(1):27-34.

22. Zorzi JL, Hage SRV. PROC - Protocolo de observação comportamental: avaliação de linguagem e aspectos cognitivos infantis. São José dos Campos: Pulso Editorial; 2004.

23. Pires SCF. A relação linguagem-cognição no trabalho com Comunicação Suplementar e/ou Alternativa com a criança com paralisia cerebral [dissertação]. São Paulo: Faculdade de Medicina da Universidade de São Paulo; 2005.
24. Bühler KEB, Flabiano FC, Mendes AE, Limongi SCO. Construção da permanência do objeto em crianças nascidas pré-termo muito baixo peso. Rev CEFAC. 2007;9(3):300-7.

25. Sato Y, Tsuga K, Akagawa Y, Tenma H. A method for quantifying complete denture quality. J Prosthet Dent. 1998;80(1):52-7.

26. Sato Y, Hamada S, Akagawa Y, Tsuga K. A method for quantifying overall satisfaction of complete denture patients. J Oral Rehabil. 2000;27(11):952-7.

27. Manubens JM, Barandiarán M, Martinez-Lage P, Francés I, Martinez C, García ML, et al. Valores del protocolo neuropsicológico GERMCIDE en una muestra de sujetos normales. Neurologia. 2005;20(4):174-9.

28. McGivney GP. Evidence-based dentistry article series. J Prosthet Dent. 2000;83(1):11-2

29. Bates E, Snyder L, Bretherton I, Volterra V. The emergency of symbols in language and action: similarities and differences. Papers and Reports on Child Development. 1979;17:106-18.

30. Bates E, Dick F. Language, gesture, and the developing brain. Dev Psychobiol. 2002;40(3):293-310.

31. Iverson JM, Longobardi E, Caselli MC. Relationship between gestures and words in children with Down's syndrome and typically developing children in the early stages of communicative development. Int J Lang Commun Disord. 2003;38(2):179-97.

32. Capone NC, McGregor KK. Gesture development: a review for clinical and research practices. J Speech Lang Hear Res. 2004;47(1):173-86.

33. Flabiano FC, Bühler KECB, Mendes AE, Limongi SCO. Quantitative and qualitative assessment of language and cognition in toddlers. In: American Speech-Language-Hearing Association - ASHA Convention, 2006. Miami. Miami Beach (FL): ASHA Convention; 2006.

34. Andrade CRF. A fonoaudiologia baseada em evidências. Einstein. 2004;2(1):61-2.

Anexo 1. Folha de registro das observações (PODCLE)

\section{Observação $n^{\circ}$}

Nome:

D.N. Idade:

Data:

\section{Cognição}

1. Aplicação de esquemas sensório-motores isolados e coordenados:

2. Deslocamento dos objetos no espaço:

\section{Permanência do objeto:}

4. Imitação de esquemas motores (com o modelo):

a. imitação de esquemas isolados visíveis no próprio corpo:

b. imitação de esquemas isolados não visíveis no próprio corpo:

c. imitação de esquemas que designam função aos objetos:

\section{Experiências com objetos novos:}

6. Uso de objetos como meios:

7. Uso de esquemas simbólicos simples (sem o modelo): a. aplicação no próprio corpo:

b. aplicação em objetos figurativos:

c. aplicação em objetos não figurativos:

8. Uso de esquemas simbólicos combinados

a. combinação de 2 ações:

b. combinação de 3 ou mais ações não ordenadas:

c. combinação de 3 ou mais ações ordenadas:

II. Linguagem Expressiva:

1. Uso de gestos dêiticos:

2. Uso de gestos representativos:

3. Produção de verbalizações acompanhadas por gestos:

4. Produção de verbalizações isoladas: 